\title{
Mechanical modeling of Interpenetrating Polymer Network reinforced acrylic elastomer
}

\author{
Arne Schmidt*ab, Andrea Bergamini ${ }^{\mathrm{a}}$, Gabor Kovacs ${ }^{\mathrm{a}}$, Edoardo Mazza ${ }^{\mathrm{ab}}$ \\ ${ }^{a}$ Swiss Federal Laboratories for Materials Testing and Research (Empa),8600 Dübendorf, \\ Switzerland; \\ ${ }^{\mathrm{b}}$ Swiss Federal Institute of Technology (ETH), Departement of Mechanical Engineering, \\ 8092 Zürich, Switzerland
}

\begin{abstract}
Interpenetrating polymer network reinforced acrylic elastomers (IPN) offer outstanding performance in free-standing contractile dielectric elastomer actuators. This work presents the verification of a recently proposed material model for a VHB 4910 based IPN [1]. The 3D large strain material model was determined from extensive data of multiaxial mechanical experiments and allows to account for the variations in material composition of IPN-membranes. We employed inflation tests to membranes of different material composition to study the materials response in a stress state different from the one that was used to extract the material parameters. By applying the material model to finite element models we successfully validated the material model in a range of material compositions typically used for dielectric elastomer actuator applications. In combination with a characterization of electro-mechanical coupling, this 3D large strain model can be used to model IPN-based dielectric elastomer actuators.
\end{abstract}

Keywords: Modeling, Hyperelastic, Multiaxial, Inflation, IPN, VHB

\section{INTRODUCTION}

Dielectric elastomer actuators (DEA) are made of soft dielectric polymer membranes that are subjected to an electrical field via compliant electrodes on opposite sides. The action of an electrical field substantially compresses the dielectric polymer in the thickness direction and expands it in the planar directions. Studies on the acrylic membrane VHB 4910 showed an increase in DEA performance by pre-straining the membrane. This effect is due to an increase in electrical breakdown strength [2] as well as geometrical effects [3]. Interpenetrating Polymer Networks (IPN) of acrylic elastomers were first presented by $\mathrm{Ha}$ et. al. [4]. They offer the performance of pre-strained acrylic elastomers without the need of a supporting structure, since the pre-strain is preserved by a force equilibrium between two polymeric networks. Kovacs et. al. [5] developed a free standing contractile actuator using IPN material. The so called stacked actuator directly uses contractive electrostatic forces instead of the passive material response in an agonist-antagonist configuration. This leads to an improved actuator performance while the absence of external pre-strain significantly enhances the reliability of the system. In general an electromechanical model suitable for FEA-simulation of actuators is needed for design optimization as well as performance prediction.

So far, different experimental approaches for the derivation of electromechanical models of DEA were presented that characterized the mechanical behavior and the electromechanical coupling either separately or combined within actuator experiments. In general, the derived electromechanical models are expected to reliably predict the behavior of actuators in different situations of stress and strain. However, in large deformation analysis difficulties are commonly observed when trying to describe the material in stress states different from the experiment used to determine material model parameters. The reason often lies in an insufficient experimental characterization of the material. In general uniaxial tensile tests alone as employed by many authors [6-9] do not provide enough information even if extended to characterize the time dependency such as in uniaxial relaxation [10-12] and creep tests [13]. To overcome these difficulties, DEA-models were determined by fitting model parameters to experimental data from actuator tests [14-16]. However this approach requires the knowledge of a suitable model of the electromechanical coupling [17;18]. Moreover,

Electroactive Polymer Actuators and Devices (EAPAD) 2010, edited by Yoseph Bar-Cohen,

Proc. of SPIE Vol. 7642, 764210 - @ 2010 SPIE - CCC code: 0277-786X/10/\$18 - doi: 10.1117/12.847505

Proc. of SPIE Vol. 7642 764210-1 
the approach of employing actuator experiments for DEA-model derivation is limited to the specific stress state, of the actuator characterized.

Recently we presented results of an extensive experimental campaign to characterize the passive mechanical response of IPN [1]. We employed uniaxial as well as equibiaxial relaxation experiments in a wide range of strain and strain rate to ensure the models applicability to different configurations. We determined a 3D large strain material model that successfully describes all experimental data. The IPN membranes tested are produced from pre-strained VHB 4910 membranes by introducing the trifunctional acrylic monomer Trimethylolpropane trimethacrylate (TMPTMA) into the membrane. This additive forms an additional polymeric network that supports part of the pre-strain. Small variations in the amount of additive will lead to a different stiffness of the second network, thus to a different level of preserved prestrain and therefore, after releasing the external pre-strain, a different membrane thickness. Since the base polymer VHB 4910 possesses a nonlinear elastic response, different levels of preserved pre-strain will lead to a different tangent modulus of the base elastomer. The mechanical behavior of the resulting material therefore strongly depends on the amount of additive. We extended our material model to account for small variations of material composition and thus effectively reduced deviations between experimental data and corresponding simulation. An investigation of the network interaction mechanisms in IPN-materials was recently presented in Zhu and Suo [19].

This work presents an extended experimental verification of the model described in [1] in order to verify the models capability of predicting the material response in different stress states. Further, a validation of the correction function accounting for the material composition is given. Since this correction function is derived from a fit to results of uniaxial relaxation experiments, the prediction of the influence of material composition has to be verified for a multiaxial stress state. We carried out membrane inflation tests with IPN-films of different material composition and applied the extended material model to axis-symmetric FEA-models of the experiments.

\section{EXPERIMENTS}

The mechanical behavior of soft elastomeric polymers such as many DEA-materials can be described using hyperelasticviscoelastic constitutive laws. The key point in such an approach is a strain energy density function describing the mechanical energy per unit volume stored in the material dependent on the strain state. Stress tensors can be derived by taking the partial derivatives of this strain energy density function with respect to the corresponding strain measure. Commonly the energy density function is highly nonlinear as well as time dependent. The determination of the in general large number of material parameters requires a considerable experimental effort to ensure an accurate prediction of the material response in different stress states. We therefore performed biaxial and uniaxial relaxation experiments for different strain values and strain rates [1]. In this work we present results of membrane inflation tests and the corresponding simulations to verify the material model derived in [1]. Membrane inflation tests were chosen because the material experiences a stress state that ranges from pure biaxial stress at the center to pure shear at the extremities. Hence the material is subject to stress states different from those we derived material parameter for.

Membrane inflation tests to study the multiaxial elastic deformation behaviour of soft rubber materials were first performed by Treloar [20] and since then applied by numerous authors [21-24]. The popularity of these experiments is based on the simplicity of the experimental set-up. A chamber filled with ideally an incompressible fluid is sealed with the circular specimen. By applying a fluid pressure the membrane deforms (Figure 1). Deformations are recorded with a camera and the fluid pressure is measured. Due to the inhomogeneity of the stress field, there is no direct way of calculating stress values without making assumptions about the local shape of the inflated membrane. Hence the derivation of stress-strain relations requires solving the inverse problem.

All experiments described in this work were conducted on a device at ETH Zurich (Figure 1). The inner radius of the clamping R was $25 \mathrm{~mm}$. The pump was operated with a constant voltage leading to a nominally constant volume flow rate of deionised water entering the chamber under the specimen. Since the stress state in the membrane depends on the material behaviour the shape of the bubble as well as the displacement $\mathrm{H}$ of the top of the bubble varies between the specimens. Nevertheless a constant volume flow rate leads to similar strain histories in all experiments. Maximal displacements $\mathrm{H}$ of the highest point of the specimen of up to $23 \mathrm{~mm}$ were achieved within $22 \mathrm{~s}$. 

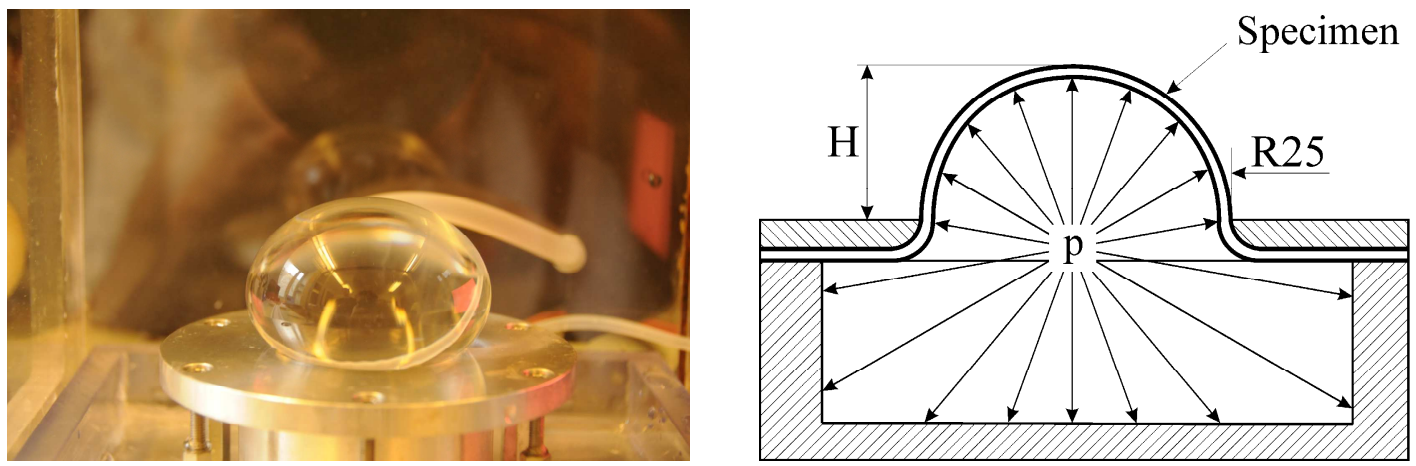

Figure 1 Membrane inflation test set-up

As already mentioned, deviations in material composition are caused by deviations in the volume ratio of both networks. This leads to membranes with a different thickness. As explained in [1] we employ the membrane thickness as variable to describe variations in material composition. To verify this approach as well as the general hyperelastic-viscoelastic material model we performed experiments with five different membranes with a thickness ranging from $66 \mu \mathrm{m}$ to $98 \mu \mathrm{m}$ obtained from VHB 4910 membranes at a pre-strain of $400 \%$. In Figure 2 the measured displacement of the top of the specimen is shown as a function of time for all five specimens. Since the same volume flow rate was applied in all experiments the different specimens experienced almost an identical deformation history. The measured pressure histories are reported for all experiments in Figure 2. Furthermore the measured pressure divided by the initial membrane thickness of each sample is given in Figure 3. We provide this relative measure of pressure since it relates to the stiffness of the material rather than to the stiffness of the structure. As it can be seen the relative pressure is quite different from sample to sample. Since the thin IPN-membranes possess a higher level of preserved pre-strain they reveal a much higher stiffness.
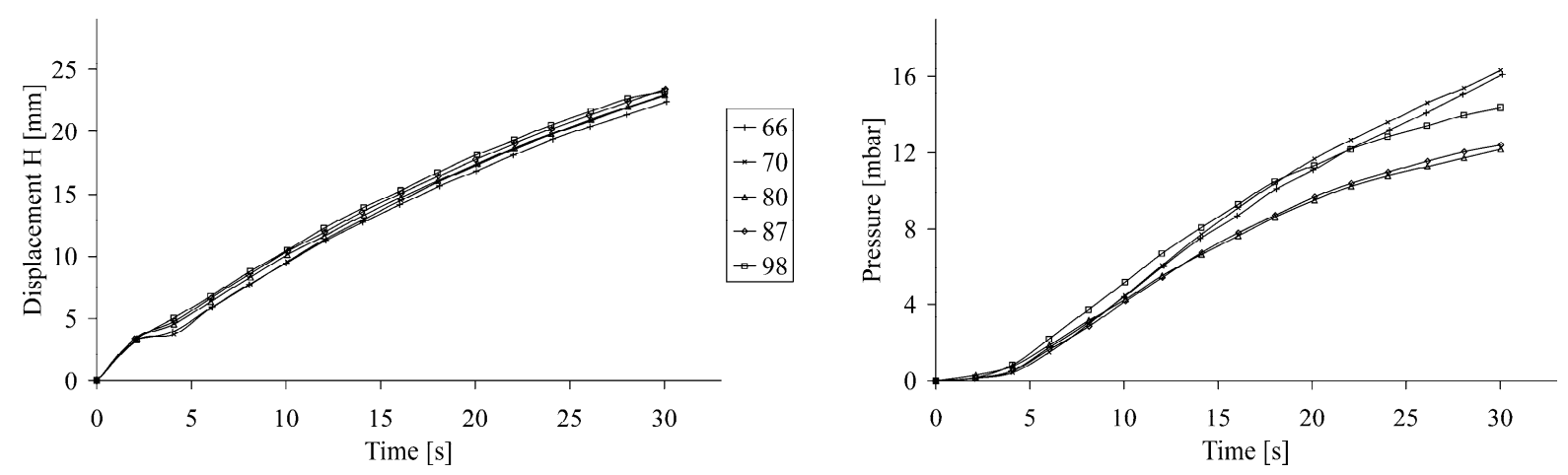

Figure 2 Displacement of the top of the bubble (left) and pressure (right) for specimens from $66 \mu \mathrm{m}$ to $98 \mu \mathrm{m}$ initial thickness 


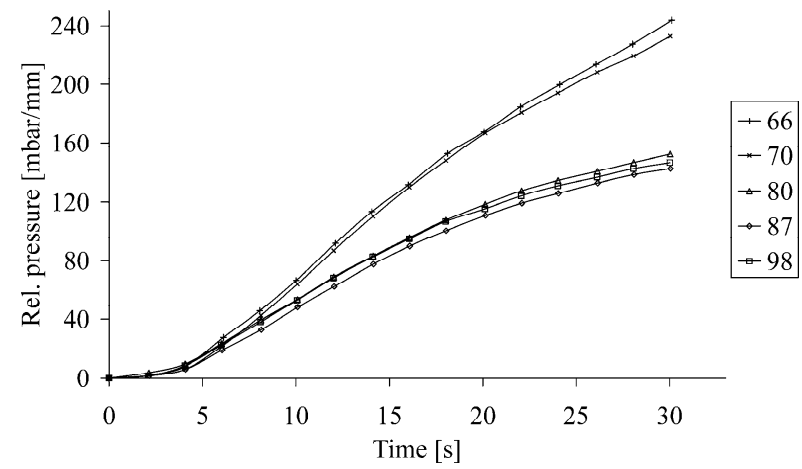

Figure 3 Relative pressure histories for specimens from $66 \mu \mathrm{m}$ to $98 \mu \mathrm{m}$ initial thickness

\section{MODELING}

\subsection{Model}

All simulations involved axis-symmetric finite element models with contact conditions. The aluminium cap of the chamber is modelled with a rigid body constraint. The fluid pressure is applied as a follower load onto the nodes at the bottom side of the specimen (Figure 4).

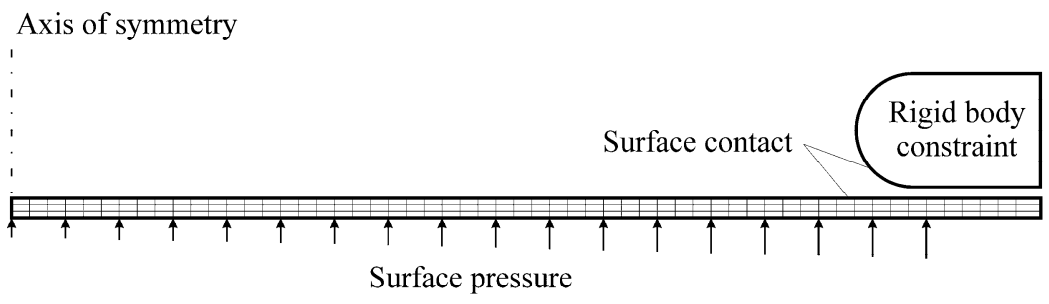

Figure 4 Finite element model of the membrane inflation test

Since the fluid pressure is measured at the bottom of the chamber, the hydrostatic pressure of the fluid needs to be subtracted to obtain the true boundary condition of the model. The straight forward approach of calculating the pressure boundary condition dependent on the vertical displacement of each node using the ABAQUS user-subroutine utracload led to a model that required a very large number of iterations to converge. We therefore first applied the measured pressure to all nodes, calculated the corresponding displacements and applied these displacement vectors to calculate the pressure boundary condition of each node of a new model. This process was repeated iteratively. The so derived displacements of the top of the specimen are shown in Figure 5 for the first three iterations. Already the results of the second and third iteration show excellent convergence. 


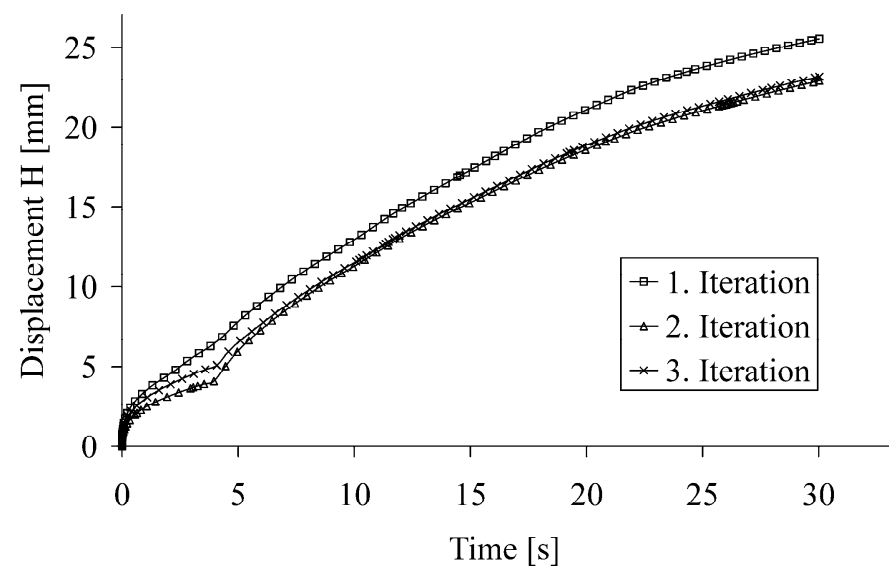

Figure 5 Evolution of calculated displacement for iterative correction of pressure boundary condition

Figure 6 shows an example of the FE calculations (membrane $70 \mu \mathrm{m}, \mathrm{H}=22 \mathrm{~mm}$ ). The color indicates the ratio between the radial and the hoop stress ranging from 1 at the center to 0.04 close to the boundaries of the sample.
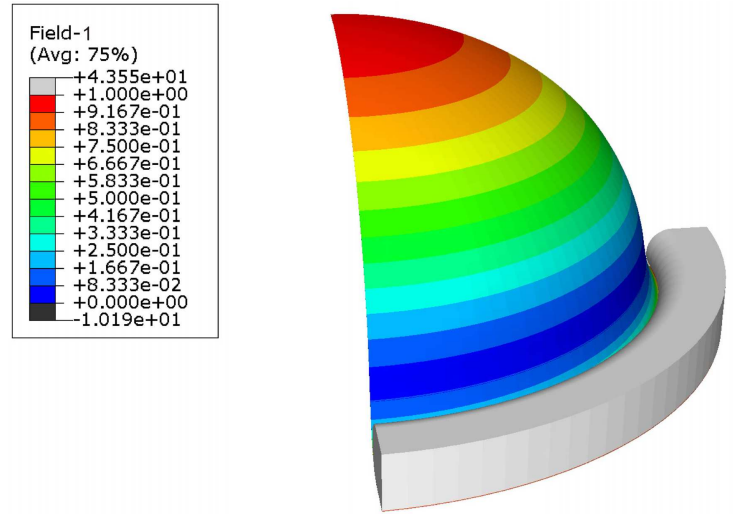

Figure 6 Radial stress divided by hoop stress in membrane of $70 \mu \mathrm{m}$ initial thickness and a displacement of $22 \mathrm{~mm}$

\subsection{Constitutive law}

For the simulation of all membrane inflation tests with IPN we employed the strain energy density function of the extended Ogden-model (1) with the parameter given in Table 1. This material model describes the time-dependent hyperelastic response of the IPN-membranes dependent on their respective undeformed membrane thickness $d$. Since the membrane thickness is resulting from the force equilibrium between both networks it can be used to describe the material composition. A detailed description of this model is given in [1].

$$
W\left(\lambda_{i}, d, t\right)=f(d) \cdot g(t) \cdot W\left(\lambda_{i}\right)=\left(\frac{d}{d_{0}}\right)^{B} \cdot\left(1-\sum_{k=1}^{4} g_{k}\left[1-e^{\left(-t / t_{k}\right)}\right]\right) \cdot\left(\sum_{j=1}^{3} \frac{\mu_{j}}{\alpha_{j}^{2}}\left(\lambda_{i}^{\alpha_{j}}-3\right)\right)
$$


Table 1 Parameter of the extended Ogden -model

\begin{tabular}{|c|c|c|c|c|c|c|c|c|}
\hline \multirow{2}{*}{$W\left(\lambda_{i}\right)$} & \multicolumn{2}{|l|}{$\mu_{1}[\mathrm{MPa}]$} & $\alpha_{1}$ & \multirow[t]{2}{*}{$\mu_{2}[\mathrm{MPa}]$} & \multirow[t]{2}{*}{$\alpha_{2}$} & \multicolumn{2}{|c|}{$\mu_{3}[\mathrm{MPa}]$} & \multirow[t]{2}{*}{$\alpha_{3}$} \\
\hline & 0.386 & & 0.206 & & & & & \\
\hline \multirow{2}{*}{$f(d)$} & $\mathrm{d}_{0}[\mathrm{~mm}]$ & & B & & & & & \\
\hline & 0.067 & & -3.76 & & & & & \\
\hline \multirow{2}{*}{$g(t)$} & $g_{1}$ & $\mathrm{t}_{1}[\mathrm{~s}]$ & $\mathrm{g}_{2}$ & $\mathrm{t}_{2}[\mathrm{~s}]$ & $g_{3}$ & $\mathrm{t}_{3}[\mathrm{~s}]$ & $\mathrm{g}_{4}$ & $\mathrm{t}_{4}[\mathrm{~s}]$ \\
\hline & 0.186 & 0.01 & 0.200 & 2.97 & 0.112 & 11.78 & 0.052 & 178.3 \\
\hline
\end{tabular}

\subsection{Calculation results}

The measured and calculated vertical displacement of the highest point of the five different membranes is shown in Figure 7. There is a very good agreement between model predictions and experimental results for the first three samples with thicknesses from 66 to $80 \mu \mathrm{m}$. This confirms that the model is capable to predict the material response in very different stress states. In fact, despite the very different relative pressure ramps shown in Figure 3 the model calculates almost the same deformation histories (Figure 7) for the three different specimens. Hence the correction function accounting for the material composition effectively reduces deviations between model and experimental data.

For the specimens with a thickness of 87 and $98 \mu \mathrm{m}$ there is a significant deviation between model and measurement. Apparently the material possesses a higher stiffness leading to smaller displacements than calculated. Since the discrepancy only concerns thick membranes, the reason for these deviations has probably to be searched in the formulation of the correction function. As already explained, the correction function was derived from results of uniaxial relaxation experiments. Therefore it might fail to correctly describe the influence of material composition in a multiaxial stress state for the case of very large membrane thickness (low volume content of the second network). Further, uniaxial experiments were only performed with membranes from 67 to $95 \mu \mathrm{m}$ thickness and thus predictions might be wrong for membranes outside this range. 


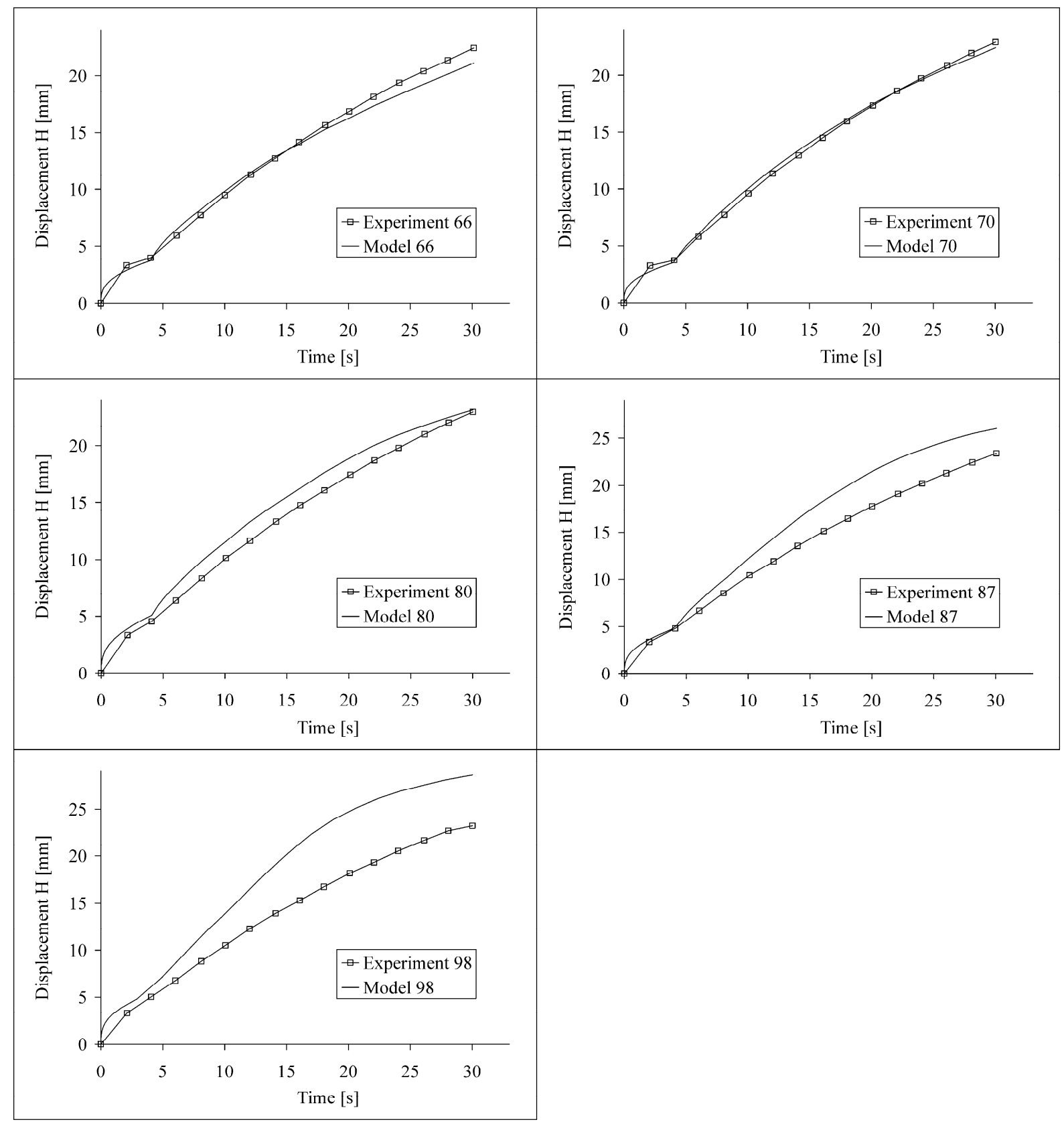

Figure 7 Measured and calculated displacement of the highest point of the specimen 


\section{CONCLUSIONS}

We employed membrane inflation tests to IPN-membranes to validate the material model proposed in [1]. These experiments were chosen since they load the material in a complex stress state thus the model is tested in a kinematic situation that is different from those used to determine the parameter of the constitutive law. Experimental data and results of axis-symmetric finite element model simulations of the experiments agree to a great extent, thus proving the validity of strain energy formulation. Tests were conducted with membranes of a different material composition. The approach of accounting for the varying material composition of IPN-membranes using a correction function based on the original membrane thickness was applied here. The prediction of this extended material model successfully describes the material behavior of the membranes with a thickness from 66 to $80 \mu \mathrm{m}$, whereas deviations are observed for the membranes of 87 and $98 \mu \mathrm{m}$ thicknesses. We therefore conclude that the model can be applied to describe the multiaxial time dependent response of IPN membranes with a level of preserved pre-strain down to $70 \%$ of the applied pre-strain of the VHB 4910 membrane.

Together with a characterization of the electro-mechanical coupling behavior of the IPN-membranes, this model can be used to simulate the electro-mechanical response of DEA of IPN-membranes.

\section{ACKNOWLEDGEMENT}

Financial support from the Swiss National Science Foundation (Project Nr. 200021-107661) is gratefully acknowledged.

\section{REFERENCES}

[1] Schmidt, A., Bergamini, A., Kovacs, G., Mazza, E., in preparation

[2] Kofod, G., Sommer-Larsen, P., Kornbluh, R. and Pelrine, R., "Actuation Response of Polyacrylate Dielectric Elastomers", Journal of Intelligent Material Systems and Structures, 14, 787-795, (2003).

[3] Kofod, G., "The static actuation of dielectric elastomer actuators: how does pre-stretch improve actuation", J. Phys. D: Appl. Phys. 41, 215405, (2008).

[4] Ha, S.M., Yuan, W., Pei, Q., Pelrine, R. and Stanford, S., "Interpenetrating networks of elastomers exhibiting $300 \%$ electrically-induced area strain", Smart Mater. Struct., 16, 280-287, (2007).

[5] Kovacs, G., Düring, L., Michel, S., Terrasi, G., "Stacked dielectric elastomer actuator for tensile force transmission", Sensors and Actuators A, 155, 299-307, (2009)

[6] Kofod, G., "Dielectric elastomer actuators", PhD-thesis, Riso-R-1286 (EN), (2001)

[7] Carpi, F., De Rossi, D., "Dielectric Elastomer cylindrical actuators: electromechanical modeling and experimental evaluation", Mat. Sci. Eng. C, 24, 555-562, (2004)

[8] Goulbourne, N., Mockensturm, N.E., Frecker, M., "A nonlinear model for dielectric elastomer membranes", Journal of Applied Mechanics, 72, 899-906, (2005)

[9] Lochmatter, P., Michel, S., Kovacs, G., "Electromechanical model for static and dynamic activation of elementary dielectric elastomer actuators", Proc. SPIE, 6168, (2006)

[10] Sommer-Larsen, P., Kofod, G., et al, "Performance of dielectric elastomer actuators and materials", Proc. SPIE, 4695, 158-166, (2001)

[11] Wissler, M., Mazza, E., "Modeling and simulation of dielectric elastomer actuators", Smart Materials and Structures, 14, 1396-1402, (2005)

[12] Ha, M.S., Wissler, M., Pelrine, R., Stanford, S., Kovacs, G., Pei, Q., "Characterization of electroelastomers based on interpenetrating polymer networks", Proc. SPIE, 6524, (2007) 
[13] Yang, E., Frecker, M., Mockensturm, E., "Viscoelastic model of dielectric elastomer membranes", Proc. SPIE, 5759, 82-92, (2005)

[14] Plante, J.S., "Dielectric elastomer actuators for binary robotics and mechatronics", Ph.D. Thesis, Department of Mechanical Engineering, Massachusetts Institute of Technology, Cambridge, MA, (2006)

[15] Plante, J.S., Dubowsky, S., "Large-scale failure modes of dielectric elastomer actuators", International Journal of Solids and Structures, 43, 7727-7751, (2006)

[16] Wissler, M., Mazza, E., "Mechanical behavior of an acrylic elastomer used in dielectric elastomer actuators", Sensors and Actuators A, 134, 494-504, (2007)

[17] Pelrine, R.E., Kornbluh, R.D., Joseph, J.P., "Electrostriction of polymer dielectrics with compliant electrodes as a means of actuation", Sensors and Actuators A 64, 77-85, (1998)

[18]Zhao, X., Suo, Z., "Electrostriction in elastic dielectrics undergoing large deformation”, Journal of Applied Physics, $104,123530,(2008)$

[19]Zhu, J., Suo, Z., "Dielectric elastomers of interpenetrating networks", Applied Physics Letters, 95, 1, (2009)

[20] Treloar, L.R.G., "Strains in inflated rubber sheet, and mechanism of bursting", Inst. Rubber Ind.-Trans., 19, 201$212,(1944)$

[21] Reuge, N., Schmidt, F.M., "Elastomer Biaxial Characterization Using Bubble Inflation Technique. I: Experimental Investigations", P. Engineering and Science, 41, 522-531, (2001)

[22] Li, Y., Nemes, J.A., "Membrane inflation of polymeric materials: Experiments and finite element simulations", Polymer Engineering and Science, 41, 1399-1412, (2001)

[23] Johannknecht, R., Jerrams, S., "Determination of non-linear, large, equal biaxial stresses and strains in thin elastomeric sheets by bubble inflation", Proc Instn Mech Engrs, 216, 233-243, (2001)

[24] Egger, J., "An Inflation Device for Testing Human Fetal Membranes, Master Thesis", Department of Mechanical and Process Engineering, Swiss Federal Institute of Technology Zuerich ETHZ, Switzerland, (2008) 